\title{
Three-Dimensional Surface Imaging in Plastic Surgery
}

\author{
Jamal Jomah* \\ Consultant Plastic Surgeon, American College of Surgeons (ACS) Governor, Saudi Arabia
}

Received: April 30, 2018; Published: May 09, 2018

${ }^{*}$ Corresponding author: Jamal Jomah, Triple Board (USA, Canada, UK), Consultant Plastic Surgeon, American College of Surgeons (ACS) Governor, MEDART \& AJMAL General Manager, American College of Surgeons (ACS) Governor, KSA, ATLS Course Director, Saudi Arabia

\begin{abstract}
Three D surface technology is used to demonstrate to the patients the expected changes possible with a particular procedure. This technology has allowed the patient and surgeon to both see the expected result at the same time on the same screen. This will help the surgeon identify the patient's expectations and be cautious about patients who have unrealistic expectations. The Surgeon can also define the steps of surgery and plan it more accurately. The goal is to improve the patient's satisfaction with this technology and minimize the revision. Three Dimensional Surface Imaging (3DSI) represents a revolution providing objective information on changes in volume or shape before and after treatment [1]. The introduction of Three Dimensional Surface Modelling (3DSM) represents a paradigm shift towards more realistic results that the patients can see and feel and appreciate from all dimensions. The aim of this paper is to describe the author's experience with 3DSI and 3DSM.
\end{abstract}

\section{Introduction}

The 3 Dimensional Surface Imaging (3DSI) has gained clinical acceptance in plastic and reconstructive surgery worldwide. Flaws are inevitable when representing a 3D structure in two-dimensional form as depth, shape and all angle are not possible. With 3DSI, it is possible to view and rotate the subject and see all views from different angles. It will allow for area and volume measurements and it is possible to appreciate visually and tactfully the changes that may result from the procedure. 3D imaging technology was first used in a clinical setting in 1944 to diagnose orthodontologic conditions. 3D surface imaging relies on triangulation, which overlays multiple images of the same object from different angles to form a 3D image, to measure surface $\mathrm{x}, \mathrm{y}$ and $\mathrm{z}$ coordinates.

\section{Aim}

The aim of this article is to review the past, present, and future directions of 3D topographic imaging in Plastic Surgery.

\section{History Of 3d}

1944 - Thalmaan used stereophogrammetry to capture the facial 3D surface and diagnose orthodontologic conditions. Tanner and Weiner attempted to standardize 3D photography by comparing anthropometric measurements to 3D measurements. 1979 -Mitchell Karlan established it in the field of Plastic Surgery, analyzing contours and documenting facial asymmetries. 1988 - Software was developed to measure distances and angles and was able to rotate the image to any angle. Cutting, it was used by Motoyosi and Russ et al. in cleft lip and proved to be accurate and clinically useful. 2002 - The use of 3D in the breast was first described. Patients undergoing unilateral breast reconstruction received quantitative measurements of breast projection, volume, and contour to determine the expander and permanent implant size for symmetry.

\section{Advantages of $3 \mathrm{dsm}$}

a) Create an edge in a competitive market.

b) Enhance the consultation to stream line the objectives and the subjective goals of the surgery.

c) Help the surgeon plan the surgical steps more precisely.

\section{D Imaging Process}
a) 3D capture
b) 3D simulation
c) 3D virtual reality
d) 3D printing

\section{D Image Acquisition}

a) Internal CT Scan MRI ICAT

b) Poorly depict external soft tissues of the body, and they are time-consuming, invasive, and cost-prohibitive. 


\section{c) External Laser Scanner Stereo photogrammetry}

\section{Present}

3D technology systems in present use include:
a) CAM3D (Enlargen, Germany)
b) C3D (Beirut, Lebanon)
c) Axis 3 (Miami, Florida, USA)
d) Canfield Scientific(Fairfield, NJ,USA)
e) Crisalix (Lau-sanne, Switzerland)
f) DI3D (Dimensional Imaging, Glasglow, Scotland)

\section{D Printers}
a) North America - MirrorMe3D
b) MENA - Maptech

\section{Which products are currently available to physicians?}

a) 3D Displays Patient specific (photobook) Standardized sets - Breast Augmentation, Rhinoplasty

\section{b) Consultation Prints Existing patients Potential patients \\ c) OR References Baseline print Intra-op reference How can 3D prints be used?}

Three Dimensional (3D) photography adds a third plane of depth to the 2 Dimensional (2D) photograph that we all used in documenting and planning our procedures. 3DSI has advantages in the form of:

I. It is possible to view and rotate the subject and see all views from different angles.

II. It will allow for area and volume measurements.

III. It is possible to appreciate visually and tactfully the changes that may result from the procedure.

\section{Method}

The 3D images are normally taken with digital camera with adequate lightning and then stored in a computer, images then manipulated using a software. The image can be captured with a camera or the surface can be scanned with a scanner but the latter would require the subject to be motionless for a longer time. The author used the Vectra H1 camera for the face and the Vectra XT for the body with the mirror image software for manipulation. A single camera is usually adequate (H1) but the 3D camera system (Vectra $\mathrm{XT}$ ) is more precise and will acquire better and larger images. Once the images are taken, they are transferred to a computer where a software stitches the images together. The problems of this systems are the cost, limited availability and resolving the shiny of transparent areas and imaging hair. With the expansion of 3D printing, the cost has substantially decreased and becoming more readily available for personal or central use. This is going to be helpful for patients' education and archiving of pre-operative and post-operative results. The technology is evolving and probably revolutionized the way we plan and document our procedures.

3D printing uses a composite powder printing process on a printer. This produces a 3 dimensional object by successively laying down the infiltrant to build a model slice by slice based on the image. This produces physical object that can be manipulated and it allows to understand the individual anatomy, can be physically held and viewed from multiple planes which is useful to teaching purposes and to better explain the proposed surgery to the patients using their own anatomy. The main limitation of the process is the cost associated with the products and with the images capturing [2].

\section{Results and Discussions}

Currently, 2 D photography is used to document, analyze and plan surgical procedures in Plastic Surgery. This tool does not represent 3 dimensional figures accurately. It lacks shaped and topographic depth. $3 \mathrm{D}$ imaging measures $\mathrm{X}, \mathrm{Y}, \mathrm{Z}$ and its coordinates and uses a triangulation concept. It is better than MRI and CT Scans which do not reflect the surfaces and surface topography and also it is less expensive and less invasive to the patient. It overlays multiple images from different planes over the same object to create a 3 D image, The use of Magnetic Resonance Imaging (MRI) is more accurate and more scientifically helpful in exact measurements however it uses screening tool that is not practical especially in cosmetic practice as MRI scans are costly and are not readily accepted by the patients. Therefore, its uses still not foreseen in the near future, The 3 Dimensional Surface Modelling (3DSM)was introduced in our clinic in the year 2014 and a comparison was conducted between the \# of cases prior to that and after that to see if it had influenced patients' decision to undergo the surgery. Subjectively, there are patients who had seen their images in 3D were more precise in their requirements and in their understanding of the outcome of the procedure. This also has modified the surgical planning and enables the surgeon to target the key areas that the patients are seeking correction (Figure 1).

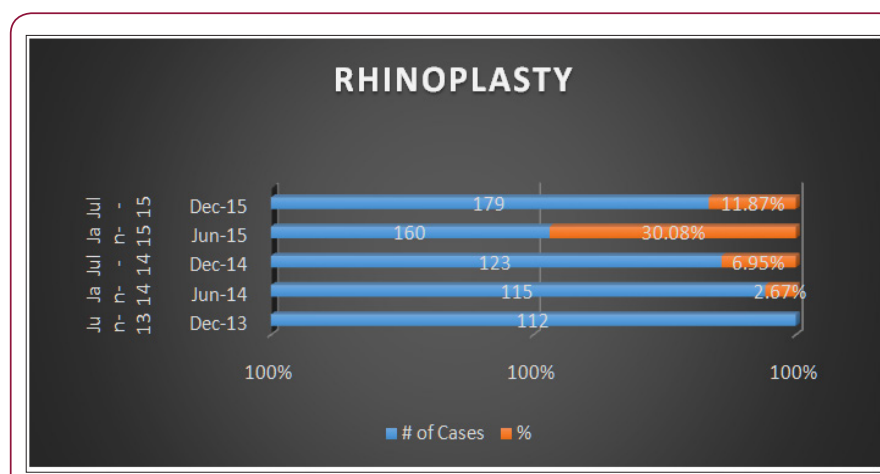

Figure 1.

\section{Conclusion}

Three Dimensional Surface Modelling (3DSM) is a useful tool in:

A. For the surgeon to understand the exact concerns of the patients and to have more precise planning of the procedure. 
B. For the patients to see the expected changes.

C. It is important to emphasize that this is only digital morphing which may not reflect the actual outcome.

\section{References}

1. Herlin et al. PRS 10.1097

2. Gillis PRS (2014) pp. 133(1).

\section{(c) (P) \\ This work is licensed under Creative Commons Attribution 4.0 License}

Submission Link: https://biomedres.us/submit-manuscript.php

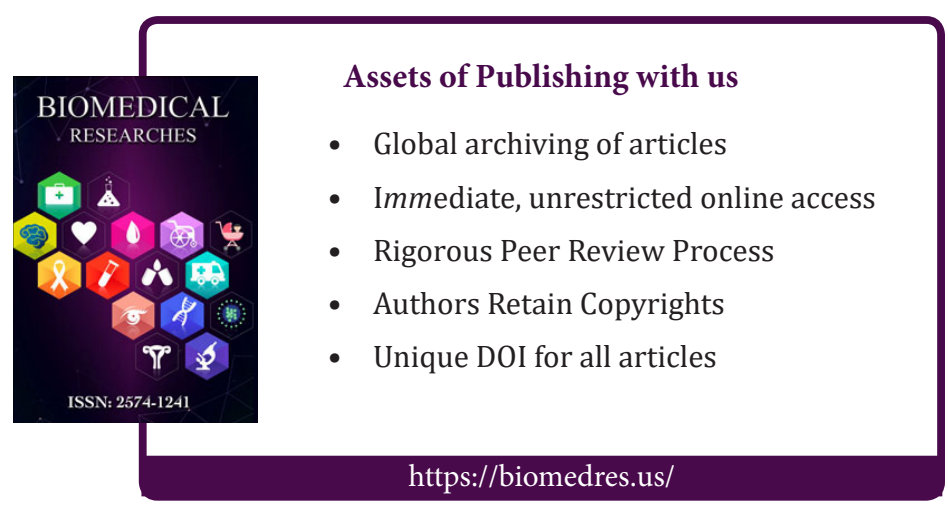

